\title{
Myocardial Protection by Blood-Based Del Nido versus St. Thomas Cardioplegia in Cardiac Surgery for Adults and Children
}

\author{
Ahmed Abdelrahman Elassal, MD, ${ }^{1,2}$ Khalid Ebrahim Al-Ebrahim, FRCSC, ${ }^{1}$ \\ Osman Osama AL-Radi, FRCSC, ${ }^{1}$ Zaher Faisal Zaher, MD, ${ }^{3}$ Ahmed Mohamed Dohain, MD, ${ }^{3,4}$ \\ Gaser Abdelmohsen Abdelmohsen, MD, ${ }^{3,4}$ Ahmed Hasan Abdulla, MS, ${ }^{1}$ Mohamed Atia Meshak, MS, ${ }^{3}$ \\ Mahmoud Akl Abdulaziz, DN, ${ }^{1}$ Mahmoud Salem Eldesouki, DN, ${ }^{1}$ Mohamed Atef Hasan,DN, ${ }^{1}$ \\ Osama Saber Eldib, MD² \\ ${ }^{1}$ Department of Surgery, Cardiac Surgery Unit, King Abdulaziz University, Jeddah, Saudi Arabia; ${ }^{2}$ Cardiothoracic Surgery \\ Department, Zagazig University, Zagazig, Egypt; ${ }^{3}$ Department of Pediatrics, Cardiology Division, King Abdulaziz University, \\ Jeddah, Saudi Arabia; ${ }^{4}$ Department of Pediatrics, Cardiology Division, Cairo University, Giza, Egypt
}

\section{ABSTRACT}

Background: St. Thomas (ST) and Del Nido (DN) cardioplegic solutions are widely used for myocardial protection during cardiac surgery. In 2016, our university hospital shifted from modified St. Thomas to Del Nido solution for both adult and pediatric cardiac surgery. This retrospective study was conducted to compare ST and DN solutions regarding surgical workflow and clinical outcome in pediatric and adult patients undergoing cardiac surgery.

Methods: We reviewed 220 patients who underwent cardiac surgery requiring cardioplegic arrest. Patients were categorized in 2 groups: ST $(n=110)$ and $\mathrm{DN}(\mathrm{n}=110)$. Each group included 60 pediatric and 50 adult patients. Demographic, intraoperative, and postoperative variables were collected.

Results: In pediatric patients, no significant difference was found between the 2 groups regarding clamping time, bypass time, need for defibrillation, inotropic score, postoperative ejection fraction (EF), period of mechanical ventilation, intensive care unit stay, or postoperative arrhythmias. One patient in the ST group required mechanical support by extracorporeal membrane oxygenation. We had 5 cases of pediatric mortality ( 3 in DN and 2 in ST, $P=.64$ ). In adult patients, significantly fewer patients in the DN group needed defibrillation than in the ST group. No significant difference was found regarding clamping time, inotropic score, or intraaortic balloon pump use. Mortality in adult patients was 6 cases (4 in ST group and 2 in DN group).

Conclusion: DN cardioplegia solution is as safe as ST solution in pediatric and adult cardiac surgery. It has comparable results of myocardial protection and clinical outcome, with superiority regarding uninterrupted surgery and lower rate of defibrillation.

Received May 28, 2020; accepted Fune 11, 2020.

Correspondence: Abmed Abdelrahman Elassal, King Abdulaziz University Hospital, Jeddah, Saudi Arabia 21589; 00966126408222 (e-mail: samalassal1434@gmail.com).

\section{INTRODUCTION}

Proper myocardial protection during cardiac surgery is crucial for favorable results. Cardioplegia remains the

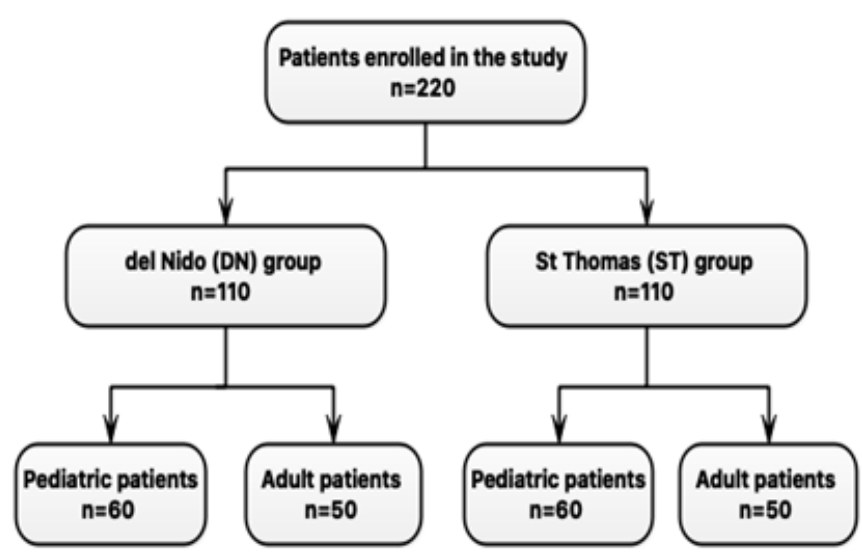

Figure 1. Patient distribution.

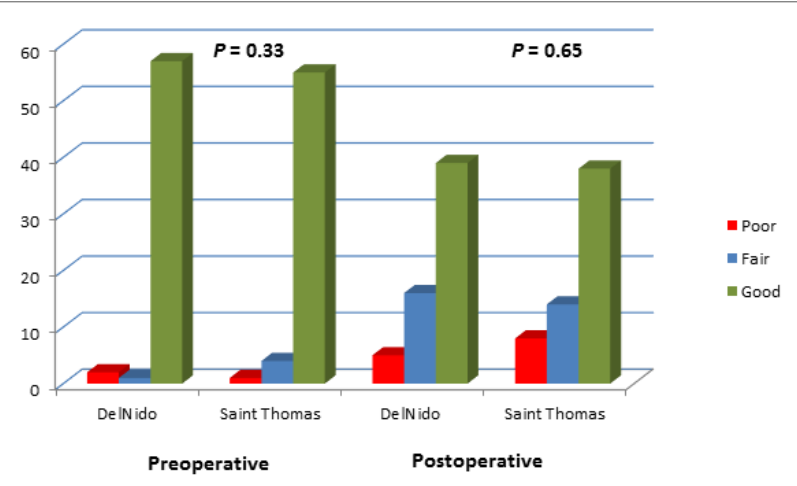

Figure 2. Preoperative and postoperative EF in pediatric patients. 
Table 1. Composition of DN Solution: Crystalloid Component

Plasma-Lyte A base solution (1000 mL) to which the following are added:

\begin{tabular}{lc}
\hline Mannitol & $20 \%, 16.3 \mathrm{~mL}$ \\
Magnesium sulfate & $50 \%, 4 \mathrm{~mL}$ \\
Sodium bicarbonate & $8.4 \%, 13 \mathrm{~mL}$ \\
Potassium chloride & $2 \mathrm{mEq} / \mathrm{mL}, 13 \mathrm{~mL}$ \\
Lidocaine & $1 \%, 13 \mathrm{~mL}$
\end{tabular}

fundamental method for myocardial protection. Thorough research has led to formulation of a variety of cardioplegic solutions and adoption of different strategies for myocardial protection [Nicolini 2003; Siddiqi 2018]. Among these solutions, St. Thomas (ST) and Del Nido (DN) are widely used. Although DN solution was originally formulated for pediatric cardiac surgery, there is an increasing interest in its use in adults. We were using ST solution in pediatric and adult cardiac surgery until 2016, when we gradually introduced DN solution. Eventually we shifted entirely to DN solution based on encouraging results of the latter and its ease of administration. We hypothesized that DN solution would provide myocardial protection equivalent or superior to that of ST solution. We carried out this retrospective study to compare the 2 cardioplegic solutions as regards efficacy and safety in adult and pediatric cardiac surgical patients.

\section{METHODS}

\section{Patient Population}

This retrospective study included 220 patients who underwent cardiac surgery requiring cardioplegia in university hospital. They were randomly selected among patients operated on between 2011 and 2019. We categorized patients into 2 groups: St. Thomas and Del Nido. Each group included 110 patients (60 children and 50 adults) (Figure 1).

We compared the 2 cardioplegic solutions in each age group separately. We excluded emergency cases, patients with myocardial infarction, reoperation for bleeding, and patients receiving mechanical circulatory support preoperatively. Demographic and clinical data were retrospectively collected including age, sex, comorbid medical conditions such as diabetes, cardiac pathology, and preoperative ejection fraction (EF). Intraoperative variables included bypass time, cross clamping (CC) time, number of cardioplegia doses, need for direct current (DC) shock, requirement for inotropes, and blood transfusions.

\section{Cardioplegia Administration}

We used blood-based crystalloid cardioplegic solutions of 2 types.

St. Thomas Solution. The blood-to-crystalloid ratio was $4: 1$ in pediatric patients and 1:4 in adults. The

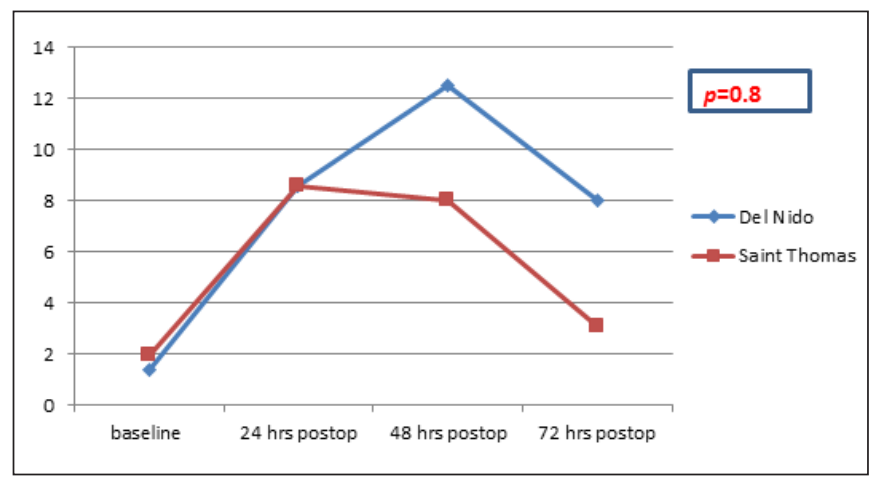

Figure 3. Pre- and postoperative troponin levels in adult patients.

crystalloid component consisted of St. Thomas' Hospital cardioplegic solution mixed with $60 \mathrm{mEq} / \mathrm{L}$ of potassium chloride and $10 \mathrm{mEq} / \mathrm{L}$ of sodium bicarbonates. The cardioplegia solution temperature was controlled with a Sorin $3 \mathrm{~T}$ Heater-cooler system. The temperature of the cardioplegic solution going to the patient was $\sim 10^{\circ}$ to $12^{\circ} \mathrm{C}$. Antegrade delivery was our preferred route of administration. We gave an initial arresting dose of $30 \mathrm{~mL} / \mathrm{kg}$ and subsequent doses of $20 \mathrm{~mL} / \mathrm{kg}$ at 20 -minute intervals or as requested by the surgeon.

DN Solution. The components of $\mathrm{DN}$ solution are shown in Table 1 . We gave a single dose of $20 \mathrm{~mL} / \mathrm{kg}$ for pediatric patients. In adult patients, we gave an induction dose of 1 liter. A second dose of $500 \mathrm{~mL}$ was given if CC time exceeded 90 minutes or if electrical activity was noticed.

\section{Outcome Measures}

Primary outcomes to measure the safety of cardioplegic solution and myocardial protection included mortality and morbidity rates, defibrillation, low cardiac output syndrome (LCOS), troponin levels, and postoperative EF. LCOS is defined as need for mechanical circulatory support device (intra-aortic balloon [IABP] or extracorporeal membrane oxygenation [ECMO]), inotropic score $>20$, or need for 2 inotropes in 4 hours. Secondary outcome measures related to workflow were bypass and clamping times, requirement for blood transfusions, period of mechanical ventilation (MV), intensive care unit (ICU) stay, and total hospital stay.

\section{Statistical Analysis}

Data were coded, entered, and analyzed using Microsoft Excel software. Data were then imported into Statistical Package for the Social Sciences (SPSS version 20.0) software for analysis. Qualitative data were presented as number and percentage, and quantitative continuous groups were presented as mean \pm standard deviation (SD) or median (interquartile range). Analysis of difference and association of qualitative variables used chi-squared test. Differences between quantitative independent groups were compared by t test, Mann-Whitney $U$ test, and logistic regression for independent predictors. $P$ values were set at $<.05$ for significant results and $<.001$ for highly significant results. 
Table 2. Preoperative, Intraoperative, and Postoperative Data in Pediatric Patients

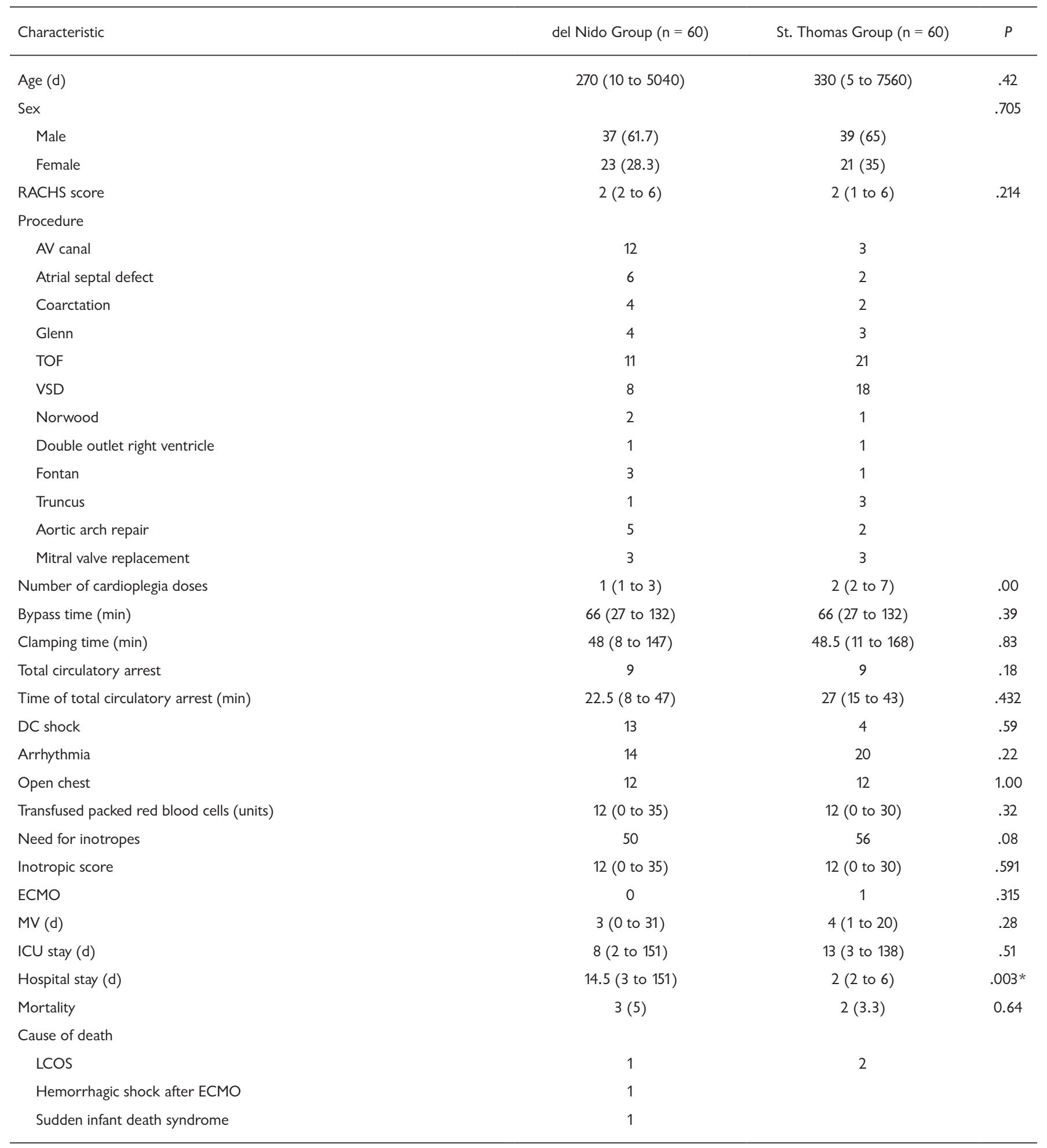

Data are median (interquartile range), n (\%), or n. *Statistically significant. 
Table 3. Predictors of LCOS

\begin{tabular}{|c|c|c|c|c|c|c|}
\hline Predictor & Control Group $(n=78)$ & LCOS Group $(n=22)$ & $P$ & Control group $(n=103)$ & LCOS group $(n=17)$ & $P$ \\
\hline Age $>60 y$ & $18(23.1)$ & $9(40.9)$ & $.03 *$ & & & \\
\hline Female sex & $14(17.9)$ & $6(27.2)$ & .13 & 35 (33.9) & $9(52.9)$ & $.039 *$ \\
\hline Preoperative EF: poor or $<40 \%$ & $14(17.9)$ & $6(27.2)$ & .13 & $1(0.97)$ & $2(11.7)$ & $.003 *$ \\
\hline Bypass time $>90 \mathrm{~min}$ & $40(51.2)$ & $15(68.1)$ & .11 & $24(23.3)$ & $3(17.6)$ & .34 \\
\hline DC shock & $34(43.5)$ & $16(72.7)$ & $.006 *$ & $9(8.7)$ & $7(41.1)$ & $.00 \dagger$ \\
\hline Type of cardioplegia & & & .053 & & & .43 \\
\hline $\mathrm{DN}$ & $35(44.8)$ & $15(68.1)$ & & $50(48.5)$ & $10(58.8)$ & \\
\hline
\end{tabular}

Data are $n(\%)$ * *Statistically significant. †Highly statistically significant.

\section{RESULTS}

\section{Pediatric Patients}

Preoperative Characteristics. Preoperative characteristics of pediatric patients are shown in Table 2. Both groups were comparable regarding age, sex, and Risk Adjustment for Congenital Heart Surgery (RACHS) score.

Intraoperative Data. Operative procedures are shown in Table 2. The most common pediatric cardiac operations were correction of tetralogy of Fallot (TOF) (26.6\%), ventricular septal defect (VSD) closure (21.6\%), and repair of atrioventricular (AV) canal (12.5\%). Intraoperative and postoperative data are shown in Table 2.

Primary Outcome. Defibrillation was needed in 9 patients $(15 \%)$ in the DN group and 7 patients $(11.6 \%)$ in the ST group, with no statistically significant difference $(P=$ $.59)$. The 2 groups were comparable regarding need for inotropes, inotropic score, and EF (Figure 2). One patient in the ST group required mechanical support by ECMO. Female sex, preoperative EF $<40 \%$, and need for DC shock were significant predictors for LCOS (Table 3). We had 5 cases of mortality ( 3 in DN and 2 in ST; $P=.64$ ). The causes of death are shown in Table 2.

Secondary Outcome. Single-dose cardioplegia was given for 49 patients $(81.6 \%)$ in the DN group versus 15 patients $(25 \%)$ in the ST group $(P=.00)$. No significant difference was found between the 2 groups regarding clamping time, bypass time, need for total circulatory arrest, period of MV, ICU stay, postoperative arrhythmias, requirement for blood transfusion, or need for delayed sternal closure. However, the total hospital stay was significantly shorter in the ST group.

\section{Adult Patients}

Preoperative Characteristics. Table 4 shows preoperative characteristics of adult patients. No statistically significant difference was found between the 2 groups regarding age, sex, hypertension, preoperative serum creatinine, or preoperative EF. Thirty-four patients in the DN group had diabetes versus 23 patients in the ST group $(P=.026)$.

Intraoperative Data. Within each group, 37 patients were operated on for isolated coronary artery bypass grafting (CABG), 11 for isolated valve surgery, and 2 for combined procedures.

Primary Outcomes. Significantly fewer patients in the DN group needed defibrillation than in the ST group (15 versus $25 ; P=.04$ ). No significant difference was found between the 2 groups regarding need for inotropes, vasoactive inotropic score (VIS), IABP use, postoperative EF, or postoperative troponin levels (Figure 3). Age $>60$ years and need for DC shock were significant predictors of LCOS (Table 3). We had 6 mortality cases (4 in the ST group and 2 in the DN group, with no significant difference; $P=.399$ ). The causes of death are shown in Table 4.

Secondary Outcomes. Single-dose cardioplegia was given in 42 patients (84\%) in the $\mathrm{DN}$ group, versus 1 patient in the ST group; $P=.00$. Bypass time was longer inthe ST group, but the difference did not reach statistical significance $(P=.24)$. The 2 groups were comparable regarding clamping time, need for blood transfusion, postoperative serum creatinine, and need for ultrafiltration. In the DN group, the mortality rate, need for inotropes, and need for defibrillation were comparable in pediatric and adult patients. However, in the ST group, adult patients had a significantly higher need for defibrillation, and pediatric patients had a higher need for inotropic support (Tables 5 and 6).

\section{DISCUSSION}

Debate still continues about the ideal myocardial protective strategy, and it is difficult to demonstrate superiority of one cardioplegic solution over another. Del Nido and St. Thomas are among the most common cardioplegic solutions 
Table 4. Preoperative, Intraoperative, and Postoperative Data in Adult Patients

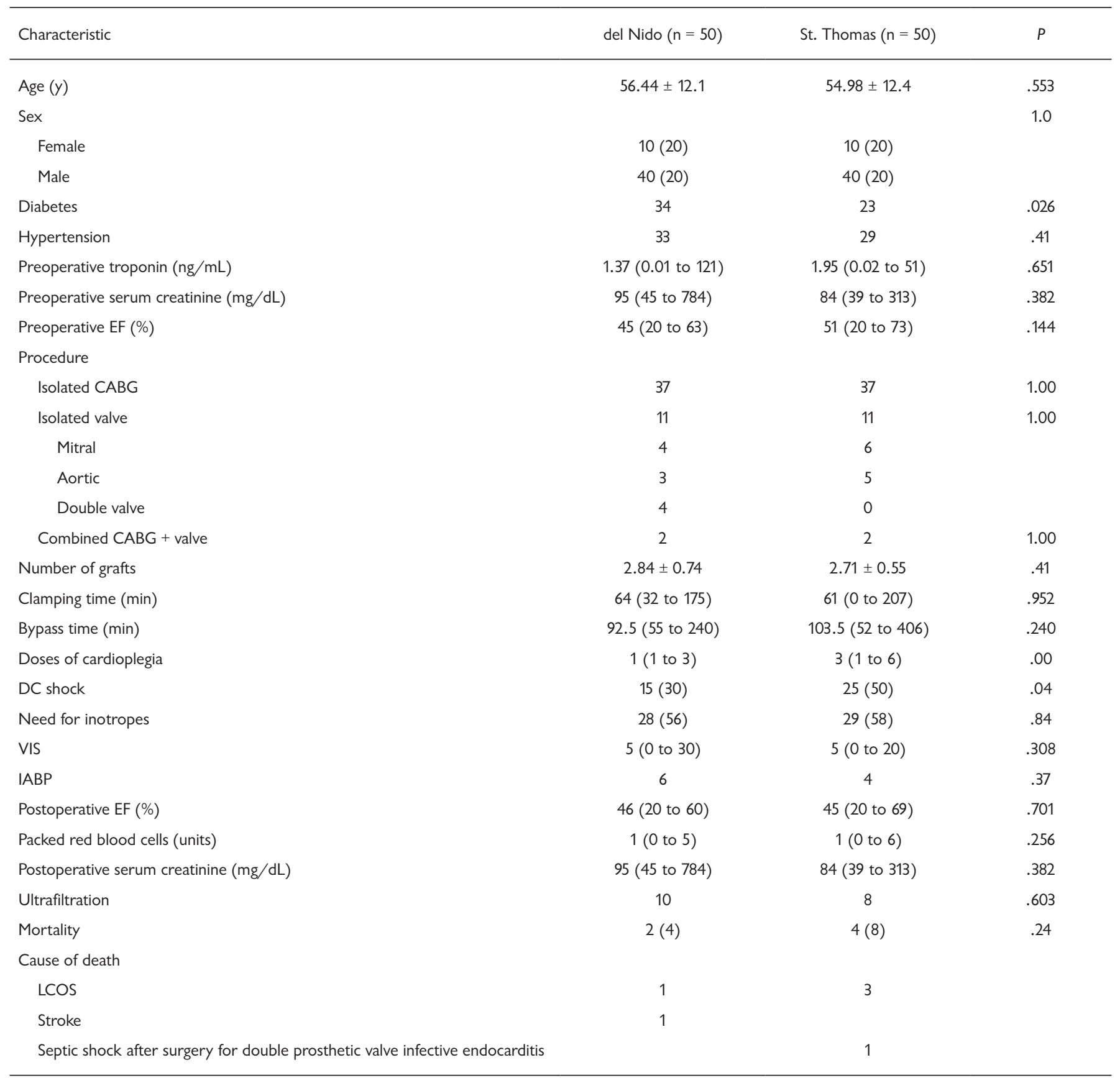

Data are mean $\pm \mathrm{SD}, \mathrm{n}(\%), \mathrm{n}$, or median (interquartile range).

currently used for myocardial protection in pediatric cardiac surgery. Both are extracellular solutions [Kotani 2013].

Starting in 2016, DN solution replaced ST solution in our protocol of cardioplegia for pediatric as well as adult patients. DN solution was originally designed to cope with requirements of immature myocardium and allow for single-dose cardioplegia in pediatric patients [Drury 2019b]. Accumulated intracellular calcium during the ischemic period causes reperfusion injury to myocardium after declamping. DN solution protects the myocardium from this harmful effect, since it contains lidocaine $\mathrm{HCl}$, which stabilizes the cell membrane and blocks $\mathrm{Na}$ ions. In addition, the $\mathrm{Mg}$ content of $\mathrm{DN}$ antagonizes calcium ions. Mannitol acts as a free oxygen radical scavenger and reduces myocardial edema because of its hyperosmolarity [McCann 2006]. 
Table 5. Outcome of DN, Comparison of Adult and Pediatric Patients

\begin{tabular}{lccc}
\hline Outcome variable & Pediatric & Adult & $P$ \\
\hline Need for inotropes & $50(83.3)$ & $28(56)$ & .12 \\
Need for defibrillation & $9(15)$ & $15(30)$ & .055 \\
Mortality & $3(5)$ & $2(4)$ & .31 \\
\hline
\end{tabular}

Data are $n(\%)$.

DN solution allows longer ischemic times between doses and hence facilitates uninterrupted surgical work [Charette 2012]. Additional doses of Del Nido are needed in patients with LV hypertrophy and in obstructive multivessel coronary artery disease, in which heterogeneous distribution of cardioplegia is a concern. Some centers use thermographic imaging as a marker for maldistribution of cardioplegia, helping decide about additional doses. We do not have this facility, so we give additional dose every 90 minutes or whenever we notice electrical or mechanical activities.

Shorter CC and cardiac bypass times were reported with Del Nido compared with St. Thomas solution. This was attributed to fewer doses needed with Del Nido, thus saving time in administering cardioplegic solutions [O'Brien 2009]. In our study, adult and pediatric patients in the DN group received fewer doses of cardioplegia. Although this did not translate to a meaningful difference in clamping or bypass times, it facilitated surgical workflow.

The total volume of cardioplegia solution is reduced when using DN. This reduces hemodilution and decreases requirement for blood transfusions. In addition, it limits myocardial edema and facilitates recovery [Sorabella 2014]. In our series, we did not find a difference in requirements for blood transfusions between the DN and ST groups.

Another advantage of use of Del Nido solution in the adult population is a lower rate of defibrillation after removal of cross clamp compared with St. Thomas solution. This minimizes cardiac injury caused by electrical defibrillation [Smigla 2014]. This has also been found to be true in pediatric patients. Buel et al [2016] reported a 6-fold decrease in rate of defibrillation with Del Nido compared with St. Thomas solution. We identified superiority of DN solution in this point in adult patients. We also found that the need for defibrillation was a significant risk factor of postoperative LCOS in pediatric and adult patients.

Measurement of troponin postoperatively was included in many series to estimate for the extent of myocardial damage. DN solution was associated with a lower rate of troponin release postoperatively. Ad et al [2018] carried out a prospective randomized trial in adult patients undergoing CABG and or valve surgery comparing DN to whole blood cardioplegia. The DN group showed lower levels of troponin, a finding that might suggest superior myocardial protection [Ad 2018]. However, other investigators could not find differences in release of biomarkers (creatine kinase $\mathrm{MB}$ and troponin I)
Table 6. Outcome of ST, Comparison of Adult and Pediatric Patients

\begin{tabular}{lccc}
\hline Outcome Variable & Pediatric & Adult & $P$ \\
\hline Need for inotropes & $56(93.3)$ & $29(58)$ & $.00^{*}$ \\
Need for defibrillation & $7(11.7)$ & $25(50)$ & $.00^{*}$ \\
Mortality & $2(3.33)$ & $4(8)$ & .161 \\
\hline
\end{tabular}

Data are n (\%).

*Statistically significant.

between DN and intermittent blood cardioplegia [Li 2018; Ucak 2019]. We do not routinely measure serum troponin after pediatric cardiac surgery; instead, we depend on measures of myocardial function such as EF and inotropic score. In adult patients, we did not find a significant difference in postoperative troponin I release between the groups.

VIS is valuable in predicting risks and outcomes after pediatric cardiac surgery. VIS $\geq 20$ in the first 24 hours postoperatively is associated with poor results [Gaies 2014]. In our series, there was no statistically significant difference in VIS between the DN and ST groups in pediatric or adult patients. However, in the ST group, the need for inotropes was significantly higher in pediatric patients than adult patients.

Drury et al [2019a] conducted a systematic review of randomized controlled trials. They reported widespread variability in the use of cardioplegia and protocols of administration. Heterogeneity of patients, interventions, and outcome measures preclude obtaining accurate results for meta-analysis. It is difficult to find definitive evidence to favor one technique over another. Thus surgeon and center preference still determines choice of cardioplegic solution [Drury 2019a, b; McCann 2006; Charette 2012; O'Brien 2009; Sorabella 2014; Smigla 2014; Buel 2016; Ad 2018; Li 2018; Ucak 2019; Gaies 2014].

In a trial to avoid heterogeneity of patients, several studies were carried out on selected groups of patients. In an isolated CABG series by O'Donnell et al [2019], the Del Nido solution group had shorter bypass and clamping times, lower inotropic support requirements, and higher return to spontaneous rhythm compared with blood cardioplegia. Yerebakan et al [2014] applied DN solution for high-risk CABG patients after acute myocardial infarction. Results were comparable to wholeblood cardioplegia in terms of mortality and morbidity. The DN group had shorter clamping and bypass times. Mick et al [2015] studied the safety of del Nido in isolated valve surgery (85 aortic valve, 110 mitral valve) in comparison with Buckberg solution (1 part crystalloid to 4 parts blood). No difference was found regarding measures of myocardial injury (highest troponin T level, postoperative EF, and inotropic score).

Del Nido solution has the advantages of lower requirement of insulin infusion and saving time and cost. A series by Vistarini et al [2017] involving patients operated on for minimal invasive aortic valve surgery showed shorter duration of mechanical ventilation in the DN group compared with blood cardioplegia administration. 
DN solution in adult cardiac surgery has been found to be safe, effective, and comparable (no superiority) to intermittent blood cardioplegia. An interesting finding in our study was that we did not identify any superiority for DN solution over ST in pediatric patients. However, adult patients in the DN group showed a higher return to spontaneous rhythm compared to the ST group. Importantly, the type of cardioplegia solution was not a significant predictor of LCOS.

\section{Limitations}

Our study has the limitations of being single center, retrospective, and not controlled. We excluded high-risk patients. Serum troponin was not measured in pediatric patients. Heterogeneity of patients and interventions might affect our results. We tried to include pediatric and adult patients to search for differences related to age and investigate whether any cardioplegia solution is superior to the other.

\section{Conclusion}

Our study showed that DN cardioplegia solution is as safe as ST solution in pediatric and adult cardiac surgery, with comparable results regarding clamping and bypass times, inotropic score, postoperative EF, troponin release (in adults), requirements of blood transfusion, and mortality. DN solution facilitated uninterrupted surgical workflow and had the advantage of a lower rate of defibrillation.

\section{ACKNOWLEDGEMENTS}

This project was funded by the Deanship of Scientific Research (DSR), King Abdulaziz University, under grant No. (DF- $826-140-1441$ ). The authors, therefore, gratefully acknowledge the .DSR technical and financial support.

\section{REFERENCES}

Ad N, Holmes SD, Massimiano PS, Rongione AJ, Fornaresio LM, Fitzgerald D. The use of del Nido cardioplegia in adult cardiac surgery: A prospective randomized trial. J Thorac Cardiovasc Surg 2018;155:1011-1018.

Buel ST, Striker CW, O’Brien JE. del Nido versus St. Thomas Cardioplegia solutions: A single-center retrospective analysis of post crossclamp defibrillation rates. J Extra Corpor Technol 2016;48:67-70.

Charette K, Gerrah R, Quaegebeur J, Chen J, Riley D, Mongero L, et al. Single dose myocardial protection technique utilizing del Nido cardioplegia solution during congenital heart surgery procedures. Perfusion 2012;27:98-103.

Drury NE, Horsburgh A, Bi R, Willetts RG, Jones TJ. Cardioplegia practice in paediatric cardiac surgery: A UK and Ireland survey. Perfusion 2019;34:125-129.
Drury NE, Yim I, Patel AJ, Oswald NK, Chong C-R, Stickley J, et al. Cardioplegia in paediatric cardiac surgery: A systematic review of randomized controlled trials. Interact Cardiovasc Thorac Surg 2019;28:144-150.

Gaies MG, Jeffries HE, Niebler RA, Pasquali SK, Donohue JE, Yu S, et al. Vasoactive-inotropic score is associated with outcome after infant cardiac surgery: An analysis from the Pediatric Cardiac Critical Care Consortium and Virtual PICU System Registries. Pediatr Crit Care Med 2014;15:529-537.

Kotani Y, Tweddell J, Gruber P, Pizarro C, Austin EH 3rd, Woods RK, et al. Current cardioplegia practice in pediatric cardiac surgery: A North American multiinstitutional survey. Ann Thorac Surg 2013;96:923-929.

Li Y, Lin H, Zhao Y, Li Z, Liu D, Wu X, et al. Del Nido cardioplegia for myocardial protection in adult cardiac surgery: A systematic review and meta-analysis. ASAIO J 2018;64:360-367.

McCann UG, Lutz CJ, Picone AL, Searles B, Gatto LA, Dilip KA, et al. Whole blood cardioplegia (minicardioplegia) reduces myocardial edema after ischemic injury and cardiopulmonary bypass. J Extra Corpor Technol 2006;38:14-21.

Mick SL, Robich MP, Houghtaling PL, Gillinov AM, Soltesz EG, Johnston DR, et al. del Nido versus Buckberg cardioplegia in adult isolated valve surgery. J Thorac Cardiovasc Surg 2015;149:626.

Nicolini F, Beghi C, Muscari C, Agostinelli A, Maria Budillon A, Spaggiari I, et al. Myocardial protection in adult cardiac surgery: Current options and future challenges. Eur J Cardiothorac Surg 2003;24:986-993

O'Brien JD, Howlett SE, Burton HJ, O'Blenes SB, Litz DS, Friesen CLH. Pediatric cardioplegia strategy results in enhanced calcium metabolism and lower serum troponin T. Ann Thorac Surg 2009;87:1517-1523.

O’Donnell C, Wang H, Tran P, Miller S, Shuttleworth P, Boyd JH. Utilization of Del Nido cardioplegia in adult coronary artery bypass grafting: A retrospective analysis. Circ J 2019;83:342-346.

Siddiqi S, Blackstone EH, Bakaeen FG. Bretschneider and del Nido solutions: Are they safe for coronary artery bypass grafting? If so, how should we use them? J Card Surg 2018;33:229-234.

Smigla G, Jaquiss R, Walczak R, Bonadonna D, Kaemmer D, Schwimer $\mathrm{C}$, et al. Assessing the safety of del Nido cardioplegia solution in adult congenital cases. Perfusion 2014;29:554-558.

Sorabella RA, Akashi H, Yerebakan H, Najjar M, Mannan A, Williams $\mathrm{MR}$, et al. Myocardial protection using del nido cardioplegia solution in adult reoperative aortic valve surgery. J Card Surg 2014;29:445-449.

Ucak HA, Uncu H. Comparison of del nido and intermittent warm blood cardioplegia in coronary artery bypass grafting surgery. Ann Thorac Cardiovasc Surg 2019;25:39-45.

Vistarini N, Laliberte E, Beauchamp P, Bouhout I, Lamarche Y, Cartier $\mathrm{R}$, et al. Del Nido cardioplegia in the setting of minimally invasive aortic valve surgery. Perfusion 2017;32:112-117.

Yerebakan H, Sorabella RA, Najjar M, Castillero E, Mongero L, Beck J, et al. Del Nido Cardioplegia can be safely administered in high-risk coronary artery bypass grafting surgery after acute myocardial infarction: A propensity matched comparison. J Cardiothorac Surg 2014;9:141. 